\title{
A non-living, effective model for microvascular training ${ }^{1}$
}

\author{
Cristina Pires Camargo', Daniel Imbassahy de Sa Bittencourt de Camara e Silva", Fauze Camargo
} Maluf'", Julio Morais-Besteiro'v , Rolf Gemperliv

'PhD, Division of Plastic Surgery, Department of Surgery, School of Medicine, Universidade de São Paulo (USP), Brazil. Intellectual and scientific content of the study, interpretation of data, statistical analysis, manuscript writing, critical revision.

"Graduate student, School of Medicine, USP, Sao Paulo-SP, Brazil. Acquisition, analysis and interpretation of data; technical procedures.

I"'Graduate student, School of Medicine, Fundação ABC, Sao Paulo-SP, Brazil. Acquisition, analysis and interpretation of data; technical procedures.

IVPhD, Division of Plastic Surgery, School of Medicine, USP, Sao Paulo-SP, Brazil. Interpretation data, critical revision.

${ }^{V}$ Full Professor and Head, Division of Plastic Surgery, Department of Surgery, School of Medicine USP, Sao Paulo-SP, Brazil. Intellectual and scientific content of the study, interpretation data, critical revision.

\section{Abstract}

Purpose: To introduce a nonliving microvascular training model based on vessels diameter and feasibility.

Methods: We dissected ten oxen tongues, and divided the pedicles into three-thirds: proximal, middle and distal. We measured the external vessels diameter in all regions. We performed a descriptive statistical analysis. Three students (two beginner level and one intermediate level) performed this training. We evaluated the confidence, according Likert scale.

Results: We dissected all oxen tongues, each tongue showed two parallel pedicles. Each pedicle was located at $1.5-2.0 \mathrm{~cm}$ from the midline. Proximal median artery and vein diameter were $3.9 \pm 0.7$, and $5.04 \pm 1.44 \mathrm{~mm}$, respectively. In the middle third, the mean artery diameter was $3.3 \pm 0.4 \mathrm{~mm}$, and the vein diameter was $3.5 \pm 0.9 \mathrm{~mm}$. The distal third showed a mean artery diameter of $2.0 \pm 0.42 \mathrm{~mm}$, and a vein diameter of $2.4 \pm 0.82 \mathrm{~mm}$. The students performed ten anastomoses. This study showed a higher confidence level (CL) $(p=0.03)$ than the pre training $C L$ assessment.

Conclusion: This study suggested a feasible non-animal model for microsurgical training process for beginners and intermediate trainees.

Key words: Microsurgery. Education. Ethics. 


\section{Introduction}

Microsurgical training needs special skills development, such as correct instrumental handling, adventectomy, which is a triangular technique for suturing. Most of the microvascular skills can be achieved in laboratory training environment ${ }^{1-3}$.

Because of ethical issues related to animal use in teaching process, most training programs use non-living animal models and simulation tasks of sutures in surgical training ${ }^{1-4}$. Stepwise learning process gives basis to the competence level on microsurgical anastomosis ${ }^{3,4}$. In our laboratory, we use the glove suture structure and then, a chicken leg. However, we observed that in some cases it was desirable to have an intermediate step in training process before chicken leg vessels anastomosis ${ }^{5-8}$. The ox tongue model can offer three different vessels' caliper to the microsurgeon student who is learning. According to the student's skills performance, a surgeon can train in the proximal, middle or distal thirds. This progressive complexity (vessels diameter) can be an intermediate step. After this training, the student can perform the chicken leg and the chicken wing training (1 $\mathrm{mm}$ artery diameter).

The aim of our study was to introduce an intermediate microvascular training model based on vessels diameter and its feasibility ${ }^{8-11}$.

\section{- Methods}

This protocol was approved by the Ethics Committee, School of Medicine Universidade de São Paulo (CEUA - 089/16).

We dissected ten oxen tongues with weight ranging from $752 \mathrm{~g}$ to $981 \mathrm{~g}$, acquired in the market. The whole anatomical structure was necessary to dissect along the dorsal face of the tongue.

\section{Model preparation}

This training required microsurgical instruments (micro scissors, microsurgical holds, and jewelry's forceps), a syringe with saline solution, and a syringe with food stain to test patency.

The structures were visualized through x4 magnification.

Two parallel and superficial pedicle composed each tongue. Both pedicles were 1.5 $-2 \mathrm{~cm}$ from the tongue midline, ventral portion. We dissected the sulcus in the muscle, and we found two veins and one artery. The veins were anterior of the artery.

We divided the vessels into three-thirds: proximal, middle and distal. We measured the arteries and veins diameters in these threethirds. Each third were indicated according to the student level experience in microvascular surgery.

\section{Microsurgical training}

In this protocol we recruited three students (two beginners and one intermediate level).

Before and the end of this training we applied a Likert scale (1= strongly disagree, 2= disagree, $3=$ somewhat disagree, $4=$ somewhat agree, $5=$ agree, $6=$ strongly agree) related to confidence $^{12}$ (Table 1 ).

Table 1 - Questions related to confidence in performing microsurgical training.

\begin{tabular}{ll}
\hline Question- I am able to do... & Grade \\
\hline Microscopy adjustment & 1 to 6 \\
Instrumental handling & 1 to 6 \\
Clamp manipulation & 1 to 6 \\
Vessels preparation for anastomosis & 1 to 6 \\
No tissue damage in the procedure & 1 to 6 \\
Complete suture & 1 to 6 \\
Perform a micro anastomoses with patency & 1 to 6 \\
\hline
\end{tabular}




\section{Surgical procedure}

For the beginner's student level the proximal and middle third suture was necessary to achieve confidence. The distal third was indicated to intermediate student level.

According to student's previous experience in microvascular end-to-end anastomosis they were designed to different third of this model. In the proximal third there were no need of magnification, the suture could be performed by 8-0 or 9-0 nylon suture. In middle third the student had to use 9-0 nylon suture. In the distal third a $x 4$ magnification were necessary and the use of $10-0$ nylon suture.

The student could use this model more than 10 times (3-4 sutures per third/ per pedicle).

After each vessels micro anastomosis, we tested the patency and leakage by injecting a mixture of saline solution and food courante using a 23-gauge needle.

\section{Statistical analysis}

We evaluated the arteries and veins diameters (mean and SD) separately according pre defined anatomical thirds.

We analyzed the confidence level between ox tongue training using Wilcoxon signed rank test. All calculations were performed by statistical software STATA version 14 (Release 14. College Station, TX: StataCorp LP).

\section{- Results}

We dissected all oxen tongues, and each tongue showed two parallel pedicles. Each pedicle was $1.5-2.0 \mathrm{~cm}$ from the midline (Figure 1).

The mean weight was $806.33 \mathrm{~g}$. The mean pedicle extension was $22.43 \mathrm{~cm}$.

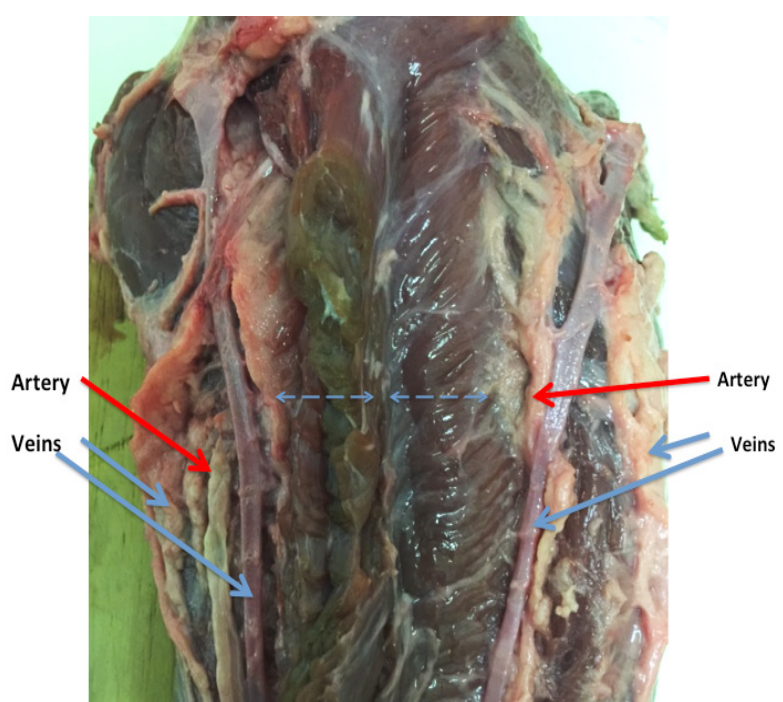

Figure 1 - The anatomical structures of ox tongue. The horizontal blue dash line measures $1.5 \mathrm{~cm}$ from the midline. The vertical blue line is the midline. The red arrows are arteries. The blue arrows are veins.

A superficial vein composed the pedicle, and due to the variability in location and diameter, we did not consider this vein for training purposes.

The next structure was a vein, located at a deeper level, and we found the artery.

Both vessels emitted several branches. We measured the external diameter of each vessels structure according to the region (proximal, middle and distal) (Table 2).

Table 2 - Diameter and standard deviation of all vessels (artery and vein) according to region (proximal, middle, distal).

\begin{tabular}{llll} 
Vessels & $\begin{array}{l}\text { Proximal } \\
\text { diameter } \\
\pm \mathrm{SD}(\mathrm{mm})\end{array}$ & $\begin{array}{l}\text { Middle } \\
\text { diameter } \\
\pm \mathrm{SD}(\mathrm{mm})\end{array}$ & $\begin{array}{l}\text { Distal } \\
\text { diameter } \\
\pm \mathrm{SD}(\mathrm{mm})\end{array}$ \\
\hline Artery & $3.9 \pm 0.7$ & $3.3 \pm 1.4$ & $2.0 \pm 0.42$ \\
Vein & $5.04 \pm 1.44$ & $3.5 \pm 0.9$ & $2.4 \pm 0.82$ \\
\hline
\end{tabular}

SD - standard deviation 
The students performed ten surgical procedures: once in the proximal third (artery and vein), three anastomoses in the middle third (artery and vein) and six anastomoses under magnification $(x 4)$ in the distal third (artery and vein).

All anastomosis were patent.

According Likert scale, this training showed a difference between pre and post questionnaire ( $p=0.03$ ) (Table 3, Figure 2).

Table 3 - Evaluation of confidence by Likert scale, before and after ox tongue training.

\begin{tabular}{lll}
\hline Trainee level & Before & After \\
\hline Beginner 1 & $26 / 42(62 \%)$ & $37 / 42(88 \%)$ \\
Beginner 2 & $25 / 42(60 \%)$ & $37 / 42(88 \%)$ \\
Intermediate & $36 / 42(86 \%)$ & $42 / 42(100 \%)$ \\
\hline
\end{tabular}

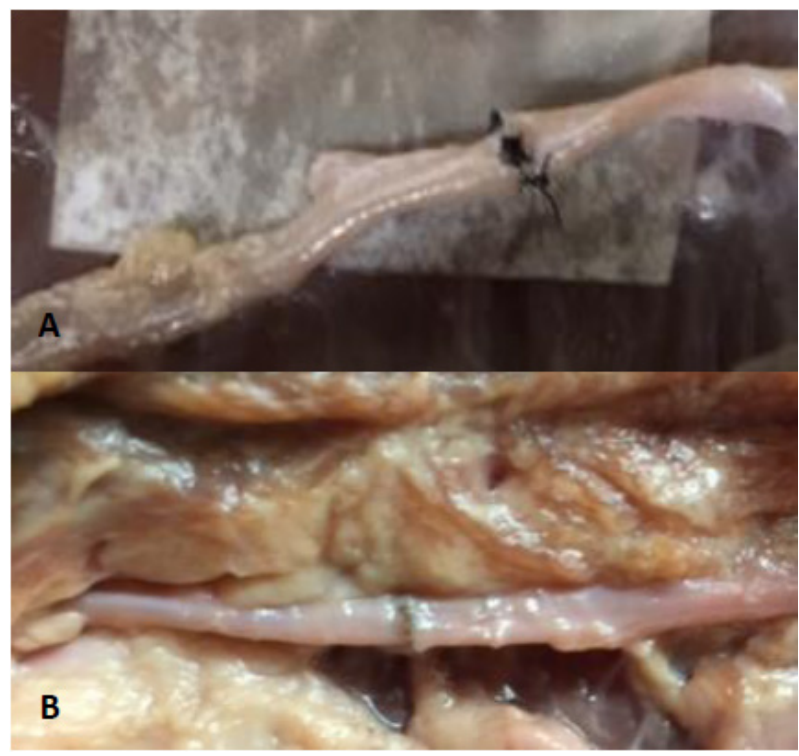

Figure 2 - Vascular anastomoses. A) First anastomosis ox tongue model-middle third. B) Fifth anastomosis ox tongue model-middle third.

\section{- Discussion}

The learning curve is essential for microsurgical training. This training demands several steps to build the curve ${ }^{8}$.

Classically, the student begins with efficient handling; the next step is glove suturing under magnification view (x4-6) and vascular anastomoses in rats ${ }^{1}$.

According to Lascar et al. ${ }^{8}$, to achieve microsurgical competence without any experience, it was necessary performing 36 end-to-end anastomosis procedures. In this paper, they showed a learning curve for eight plastic surgeon students. This training used 134 rats, an average of 16.5 rats per resident. Nowadays, the use of animals has ethical issues. Thus, alternative non-living animal models are desirable.

As previously cited, all surgical skills acquisition demands repetition. For this reason, a training protocol based on step-wise crescent complexity is desirable ${ }^{8,9}$.

Some microsurgical skills were based on low-fidelity models, such as a plastic/silicon tubes, leafs, and needles. All these activities focus on training a particular skill: precision, hand motion, and spatial motor skill ${ }^{8,9}$. However, a high fidelity model as the nonliving anatomical structure is important for the student to simulate $i t^{10,11}$.

Most of the training protocol begins with a glove suture procedure, then to a chicken leg and finally, to chicken wing anastomosis.

In our educational practice, we were able to observe that mainly for beginners, there was a difficulty to operate the chicken leg model after the glove structure task. Considering this obstacle, we proposed an intermediate level of anastomosis model to prepare our students for more advanced activities.

An ox tongue is easy to purchase, and it is low-cost. This anatomical structure presents two parallels pedicles. The diameter of this vessels decreases from the proximal third to the distal third of the tongue. Depending on the resident's expertise, one could practice in proximal or distal vessels. The artery 
diameter ranged from $3.9 \mathrm{~mm}$ to $2.0 \mathrm{~mm}$, and the vessel diameter ranged from $5.04 \mathrm{~mm}$ to $3.06 \mathrm{~mm}$. Moreover, the student can practice anastomosis in a long extension $(22.43 \mathrm{~cm})$ and redo the suture more than three times.

We tested this model in three students, two beginners and one intermediate level. The proximal third was easy to handle, the students performed the task without any magnification. The middle third showed more complexity, the diameter was $3.3 \mathrm{~mm}$ per artery and 3.4 per vein. This diameter was similar of epigastric vessels ${ }^{13}$

The next step, distal vessels needed magnification, this training showed vessels with similar diameter of internal mammary artery and anterior cerebral artery $(1.9-2.6 \mathrm{~mm})^{14}$ or anterior cerebral artery ${ }^{15}$ and facial artery $(2.83 \pm 0.77 \mathrm{~mm})^{16}$.

After this procedure, it would be easier for the resident to train in a chicken leg vessel (average diameter of $1.3 \mathrm{~mm}$ ) and, in a chicken wing model (average diameter of $1.0 \mathrm{~mm}$ ).

We submitted three students (two beginners and one intermediate level) to test this model. Each resident performed three to five anastomoses in the same ox tongue unit. This training model showed a difference in confidence between pre and post-training program $(p=0.003)$.

The strength of our study was to present a feasible, low-cost non-living animal model. The limitation of our study was the small number of students' evaluation in this micro surgical training model. After describing this model, we will apply more students to assessment of learning curve and the effectiveness of this intermediate step in the complete micro surgical training. Another possible study is to apply a pump to mimic blood flow, to test the patency of the anastomosis.

\section{Conclusions}

This new model showed adequate vessels diameter for the microsurgical training of beginners and intermediate student levels. Also, it could be part of a complete step-wise training program using non-living animal models.

\section{References}

1. Shurey S, Akelina Y, Legagneux J, Malzone G, JigaL, Ghanem AM. Ratmodel in microsurgery education: classical exercises and new horizons. Arch Plast Surg. 2014;41(3):201-8. doi: 10.5999/aps.2014.41.3.201.

2. Hong JW, Kim YS, Lee WJ, Hong HJ, Roh TS, Song SY. Evaluation of the efficacy of microsurgical practice through time factor added protocol: microsurgical training using nonvital material. J Craniofac Surg. 2010;21(3):876-81. PMID: 20485073.

3. Aurich LA, Silva Junior LFM, Monteiro FMR, Ottoni AN, Jung GS, Ramina R. Microsurgical training model with nonliving swine head. Alternative for neurosurgical education. Acta Cir Bras. 2014;29(6):405-9. doi: 10.1590/S0102-86502014000600010.

4. Couceiro J, Castro R, Tien, H, Ozyurekoglu T. Step by step: microsurgical training method combining two nonliving animal models. J Vis Exp. 2015;(99):e52625. doi: 10.3791/52625.

5. Erel E, Aivenibe B, Butler PE. Microsurgery simulators in virtual reality: a review. Microsurgery. 2003;23(2):147-52. PMID: 12740888.

6. Jeong HS, Moon MS, Kim HS, Lin HK, Yi SI. Microsurgical training with fresh chicken legs. Ann Plast Surg. 2013;70(1):57-61. PMID: 22156886.

7. Kim BJ, Kim ST, Jeong YG, Lee $W H$, Lee $\mathrm{KS}$, Paeng $\mathrm{SH}$. An efficient microvascular anastomosis training model based on chicken wings and simple instruments. J Cerebrovasc Endovasc Neuer. 2013;15(1):205. doi: 10.7461/jcen.2013.15.1.20.

8. Lascar O, Toti DE, Simica A, Cortana S, Stefanescu A, Bratianu R, Udrescu G, Calcaianu N, Zamfirescu DG. Training program and learning curve in experimental microsurgery during the residency in plastic surgery. Microsurgery. 2007;27:263-7. PMID: 22421490.

9. Hino A. Training in microvascular surgery using a chicken wing artery. Neurosurgery. 
2003;52:1495-7. PMID: 12762899.

10.Dumont L-A, Martinot-duquennoy V, Hubert T, Guerreschi P. The "double clock" or how to learn microsurgery without animal. Ann Chir Plast Esthet. 2011 Dec;56(6):555-7. doi: 10.1016/j.anplas.2011.08.014.

11.Ramachandran S, Ali G, Simon M. Microsurgical skill acquisition learning curves are normally distributed: implications for postgraduate career selection and surgical training curriculum design. Plastic Reconstr Surg. 2012;130(5S-1):102. doi: 10.1097/01. prs.0000421817.85476.af.

12.Clanton J, Gardner A, Cheung M, Mellert L, Evancho-Chapman M, George L. The relationship between confidence and competence in the development of surgical skills. J Surg Educ. 2014;71(3):405-11. doi: 10.1016/j.jsurg.2013.08.009.

13. Milgaltier E, Peral JM, LAsjs H, Elami A, Baker ED, Buckberg GD. The inferior epigastric arteries as coronary bypass conduits. Size, preoperative duplex scan assessment of suitability, and early clinical experience. J Thorac Cardiovasc Surg. 1992;103(3):463-5. PMID: 1532038.

14.Otsuka F, Yahagi K, Sakakura K, Virmani R. Why is the mammary artery so special and what protects it from atherosclerosis? Ann Cardiothorac Surg. 2013;2(4), 519-26. doi: 10.3978/j.issn.2225-319X.2013.07.06.

15.Stefani MA, Schneider FL, Marrone AC, Severino AG, Jackowski AP, Wallace MC. Anatomic variations of anterior cerebral artery cortical branches. Clin Anat. 2000;13(4):231-6. PMID: 10873213.

16.Jiang $\mathrm{GH}$, Yan $\mathrm{JH}$, Lin $\mathrm{CL}$, Huang $\mathrm{Y}$, Wen $\mathrm{H}$, Li WM. Anatomic study of the facial artery using multislice spiral CT angiography. Nan Fang Yi Ke Da Xue Xue Bao. 2008;28(3):4579. PMID: 18359713.

\section{Correspondence:}

Cristina Pires Camargo

Avenida Brigadeiro Luis Antonio, 4161

01402-002 São Paulo - SP Brasil

cristinacamargo@usp.br

Received: Aug 23, 2017

Review: Oct 25, 2017

Accepted: Nov 22, 2017
Conflict of interest: none

Financial source: none
${ }^{1}$ Research performed at Division of Plastic Surgery, Department of Surgery, School of Medicine, Universidade de São Paulo (USP), Brazil. 